\title{
Die Beckenbodenspezialistin
}

BÄRBEL JUNGINGER Schon seit 15 Jahren befasst sich die Physiotherapeutin Bärbel Junginger mit der Rehabilitation des Beckenbodens und hat an vielen Studien dazu mitgewirkt. Ihr Credo: Nicht so viel Wert auf Kräftigung legen, sondern auf motorische Kontrolle.

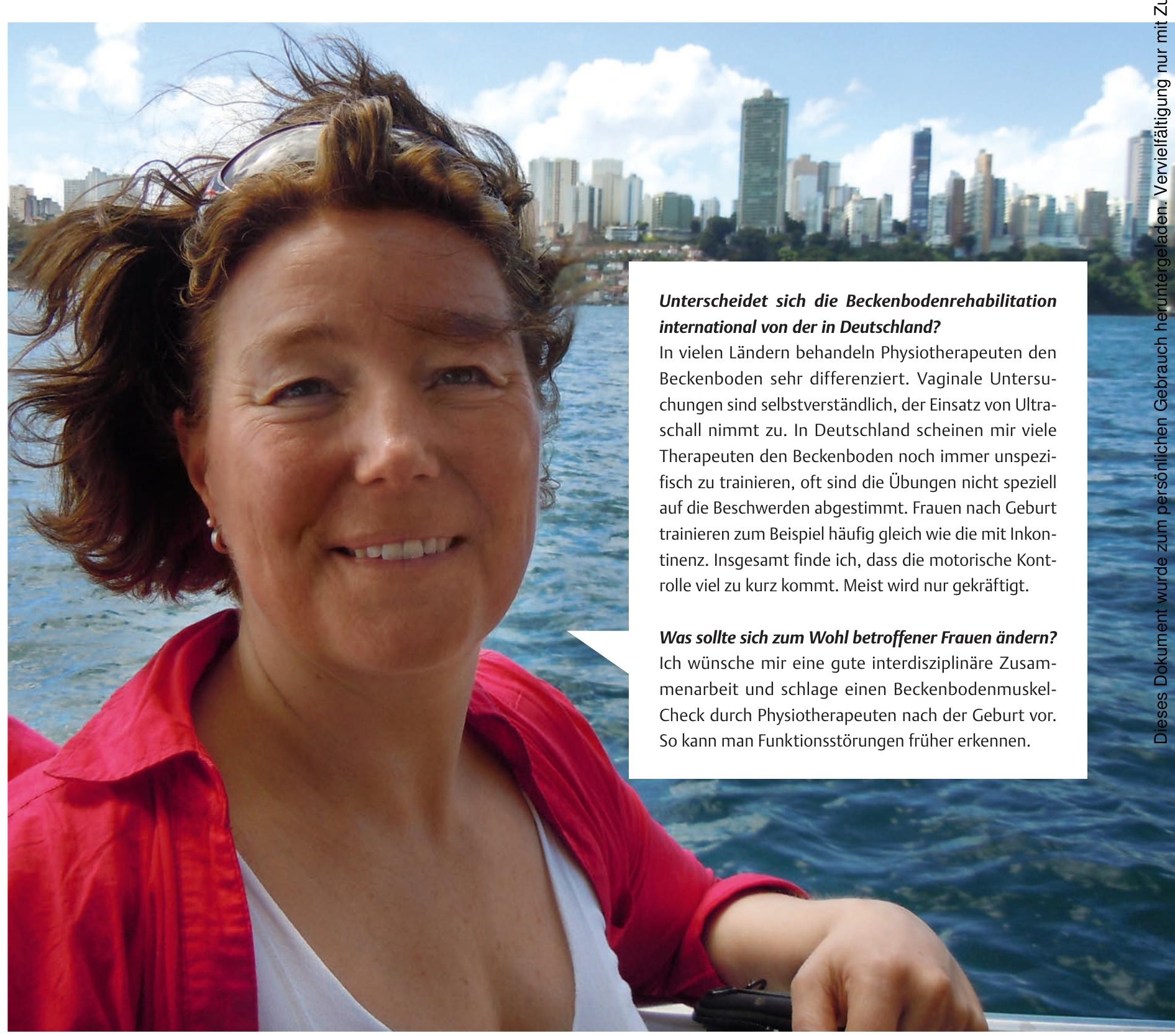




\section{Den Blasenhals stabilisieren}

\section{Bärbel Junginger...}

... lebt in Berlin und ist wissenschaftliche Mitarbeiterin im Beckenbodenzentrum der Charité. Zudem behandelt sie Patienten und unterrichtet international in verschiedenen Studiengängen. Seit über 15 Jahren hat sie sich auf den Beckenboden und dessen ultraschallgestützte Therapie spezialisiert. Gemeinsam mit der Uro-Gynäkologin PD Dr. Kaven Baessler entwickelte sie ein evidenzbasiertes Konzept zur Behandlung von Beckenbodenstörungen, das „Junginger-Baessler-Konzept“. Neben selbst entwickelten und validierten Fragebögen setzten sie dabei den dynamischen rehabilitativen Ultraschall (DRUS) zur Diagnostik und als Biofeedbackverfahren ein. Damit können Patientinnen selbst kontrollieren, ob sie die richtigen Muskeln anspannen. Zurzeit schreibt Bärbel Junginger ihre Masterarbeit in Australien bei Prof. Paul Hodges. Sie untersucht, wie Bauch- und Beckenbodenmuskulatur bei unterschiedlichen Kontraktionsformen zusammenwirken und wie sich diese Kontraktionsformen auf intraabdominellen Druck und Blasenhalsposition auswirken. Bärbel Junginger kommt durch ihre Arbeit viel herum, genießt die Auslandsaufenthalte sehr und plant stets Zeit ein, um Land und Leute kennenzulernen.

\section{Das Forschungsfeld}

Funktionsstörungen des Beckenbodens kommen häufig vor. Nicht nur ältere Frauen sind davon betroffen, auch viele jüngere Frauen nach Geburten fühlen sich durch Symptome wie Inkontinenz und Beckenbodensenkungen in Sport und Sexualität eingeschränkt. Primäres Ziel der Beckenbodenrehabilitation ist eine Steigerung der Lebensqualität über die Verbesserung beziehungsweise den Erhalt der Blasen-, Darm- und Sexualfunktion. Physiotherapeuten setzen dazu häufig auf eine allgemeine Beckenbodengymnastik, deren langfristige Wirksamkeit allerdings oft unbefriedigend ist. Bärbel Jungingers Ziel ist es, die Evidenzbasierung in der Beckenbodenrehabilitation voranzutreiben. Dazu hat sie in den vergangenen Jahren zahlreiche Forschungsergebnisse zusammengetragen und selbst Untersuchungen durchgeführt. Aktuell wirkt sie an einer von der Deutschen Forschungsgemeinschaft geförderten Studie mit, in der erstmals die Beckenbodenaktivierungsstrategien im Alltag gesunder Frauen untersucht und mit denen von Frauen mit Harninkontinenz verglichen werden. Dabei will man herausfinden, welche Mechanismen zur Kontinenzsicherung bei den betroffenen Frauen versagen. In einem weiteren Schritt soll dann die Wirksamkeit des eigenen „Junginger-Baessler-Konzeptes“ überprüft werden. Dieses basiert auf der Erkenntnis, dass zur Sicherung der Harnkontinenz der Blasenhals muskulär stabilisiert werden muss. Inkontinente Frauen können das offenbar nicht ausreichend.

Die folgenden Ergebnisse und Schlussfolgerungen sind eine Zusammenfassung aller Untersuchungen, an denen Bärbel Junginger bisher mitgewirkt hat. Im Zentrum dieser Studien stand die Frage, wie Beckenbodenkontraktionen erfolgen müssen, damit der Blasenhals effektiv stabilisiert wird.

\section{Bisherige Ergebnisse}

Bärbel Junginger hat bisher herausgefunden, dass ...

> zur Sicherung der Blasenposition beim Niesen oder Husten eine Präkontraktion der Beckenbodenmuskulatur erforderlich ist.

$>$ der intraabdominelle Druck nur gering ansteigt und der Blasenhals wie gewünscht stabilisiert wird, wenn man den Beckenboden submaximal kontrahiert.

> auch eine gezielte selektive Aktivität des M. transversus abdominis den intraabdominellen Druck kaum erhöht und mithilft, den Blasenhals zu stabilisieren.

> herkömmliche Beckenbodenübungen bei gesunden Frauen dagegen teilweise dazu führen, dass der Blasenhals absinkt. Somit sind diese Übungen vermutlich nicht für die Beckenbodenrehabilitation geeignet.

$>$ das Training der motorischen Kontrolle wirksam ist: Nach sieben Monaten gaben 67\% der Frauen mit Stressinkontinenz an, dass sich ihre Beschwerden verbessert haben, bei denen mit Dranginkontinenz waren es $78 \%$.

\section{Fazit}

Zusammenfassend kann Bärbel Junginger festhalten, dass ...

> eine effektive Beckenbodenrehabilitation nicht die Muskelkraft, sondern die motorische Kontrolle verbessern sollte.

> dazu eine gezielte, submaximale Anspannung des Beckenbodens unter Kokontraktion des M. transversus abdominis erforderlich ist.

> Frauen dabei begleitet werden müssen, diese submaximale Kontraktion in den Alltag zu integrieren.

Eva Trompetter

$\rightarrow$ Publikationen unter www.physiotherapie-junginger.de > „Publikationen“ 